\title{
Recent Developments in Few-Nucleon Systems
}

\author{
A. Kievsky ${ }^{\mathrm{a}}$, M. Viviani ${ }^{\mathrm{a}}$, L.E. Marcucci ${ }^{\mathrm{a}}$ and S. Rosati ${ }^{\mathrm{a}}$ \\ astituto Nazionale di Fisica Nucleare, and Physics Department, Universita' di Pisa, Via \\ Buonarroti 2, 56100, Italy
}

\begin{abstract}
$N-d$ elastic scattering is studied at different energies using one of the modern NN interactions, the Argonne $v_{18}$ which explicitly includes the magnetic moment interaction between two nucleons. This interaction, which has been often neglected in the description of the few-nucleon continuum, produces sizable modifications in some elastic observables. Its effects, as well as those produced by the Coulomb potential, are analyzed as a function of energy. The magnetic moment interaction produces appreciable effects in $p-d$ scattering at low energies but they are very small above $10 \mathrm{MeV}$. Above $65 \mathrm{MeV}$ Coulomb effects can be observed only in specific observables as for example $T_{21}$.
\end{abstract}

\section{Introduction}

The three- and four-nucleon systems are excellent testing grounds for the nuclear interaction. The last generation $N N$ interactions can be used to calculate $3 N$ and $4 N$ bound and scattering states and, from a comparison to the experimental data, important conclusions about the capability of those interactions to reproduce the dynamics can be extracted. It is widely accepted that the potential energy in a few-nucleon system consists of a sum of the pairwise $N N$ interaction and a term including a pure three-nucleon interaction. This last term is not very well known and, in general, its strength is fixed so as to reproduce the experimental $A=3$ binding energy. With the recent advances in the solution of the $3 N$ and $4 N$ continuum, the possibility of using scattering data to improve our knowledge of the three-nucleon interaction is at present feasible. Because of this, a correct treatment of the complete electromagnetic interaction in the description of fewnucleon reactions is required. In fact, Coulomb effects and three-nucleon force effects are very often of the same size. Moreover, the magnetic moment (MM) interaction between two-nucleons can give sizable contributions to some polarization observables.

In the present contribution $N-d$ scattering is studied using the Argonne $v_{18}$ (AV18) NN potential [1] plus the Urbana IX (UR) three-nucleon potential [2] and electromagnetic terms as the Coulomb and magnetic moment interactions. Different observables are considered such as the differential cross section and vector and tensor analyzing powers. A comparison to recent high-quality measurements is performed. 


\section{The $N-d$ Transition Matrix including the MM Interaction}

Following the notation used in the determination of the AV18 potential, all modern $N N$ potentials can be put in the general form

$v(N N)=v^{E M}(N N)+v^{\pi}(N N)+v^{R}(N N)$.

The short range part $v^{R}(N N)$ of these interactions includes a certain number of parameters (around 40), which are determined by a fitting procedure to the $N N$ scattering data and the deuteron binding energy $(\mathrm{BE})$, whereas the long range part is represented by the one-pion-exchange potential $v^{\pi}(N N)$ and the electromagnetic potential $v^{E M}(N N)$.

The AV18 potential includes the same $v^{E M}(N N)$ used in the Nijmegen partial-wave analysis except for short-range terms and finite size corrections. The $v^{E M}(p p)$ consists of the one- and two-photon Coulomb terms plus the Darwin-Foldy term, vacuum polarization and MM interactions. The $v^{E M}(n p)$ interaction includes a Coulomb term due to the neutron charge distribution in addition to the MM interaction. Finally, $v^{E M}(n n)$ is given by the MM interaction only. All these terms take into account the finite size of the nucleon charge distributions. Explicitly the long range part of the $N N$ magnetic moment interaction reads:

$$
\begin{aligned}
v_{M M}(p p) & =-\frac{\alpha}{4 M_{p}^{2}}\left[\mu_{p}^{2} \frac{S_{i j}}{r^{3}}+\left(8 \mu_{p}-2\right) \frac{\mathbf{L} \cdot \mathbf{S}}{r^{3}}\right], \\
v_{M M}(n p) & =-\frac{\alpha \mu_{n}}{4 M_{n} M_{p}}\left[\mu_{p} \frac{S_{i j}}{r^{3}}+\frac{2 M_{p}}{M_{n p}} \frac{(\mathbf{L} \cdot \mathbf{S}+\mathbf{L} \cdot \mathbf{A})}{r^{3}}\right], \\
v_{M M}(n n) & =-\frac{\alpha \mu_{n}^{2}}{4 M_{n}^{2}} \frac{S_{i j}}{r^{3}} .
\end{aligned}
$$

$M_{p}\left(M_{n}\right)$ is the proton (neutron) mass and $M_{n p}$ is the $n-p$ reduced mass. The MM interaction presents the usual $r^{-3}$ behavior and has an operatorial structure. In the $n p$ case, the last term includes an asymmetric force (proportional to $\mathbf{A}=\left(\boldsymbol{\sigma}_{i}-\boldsymbol{\sigma}_{j}\right) / 2$ ) which mixes spin-singlet and spin-triplet states.

For $A=2$ the contribution of the MM interaction to the the scattering amplitude has been extensively studied [ [3, 4]. It has been shown that due to its $r^{-3}$ behavior the scattering amplitude results in a slow convergent series whose leading terms can be summed analytically. A similar analysis can be performed for $N-d$ scattering. Accordingly, the $N-d$ transition matrix $M$ is written as

$$
\begin{aligned}
M_{\nu \nu^{\prime}}^{S S^{\prime}}(\theta) & =f_{c}(\theta) \delta_{S S^{\prime}} \delta \nu \nu^{\prime}+f_{M M}(\theta)+\frac{\sqrt{4 \pi}}{k} \sum_{L L^{\prime}}^{L_{\max }} \sum_{J} \sqrt{2 L+1}(L 0 S \nu \mid J \nu)\left(L^{\prime} M^{\prime} S^{\prime} \nu^{\prime} \mid J \nu\right) \\
& \times \exp \left[i\left(\sigma_{L}+\sigma_{L^{\prime}}-2 \sigma_{0}\right)\right]^{J} T_{L L^{\prime}}^{S S^{\prime}} Y_{L^{\prime} M^{\prime}}(\theta, 0) .
\end{aligned}
$$

The matrix $M$ is a $6 \times 6$ matrix corresponding to the projections of the two possible couplings of the spin of the deuteron $S_{d}=1$ and the spin $1 / 2$ of the third particle to $S=$ $1 / 2$ or $3 / 2$. The quantum numbers $L, L^{\prime}$ represent the relative orbital angular momentum between the deuteron and the third particle and $J$ is the total angular momentum of 
the three-nucleon scattering state. ${ }^{J} T_{T T^{\prime}}^{S S^{\prime}}$ are the $T$-matrix elements corresponding to a Hamiltonian containing nuclear plus Coulomb plus MM interactions. In the above equation $f_{c}$ is the Coulomb amplitude:

$f_{c}(\theta)=\sum_{L=0}^{\infty}(2 L+1)\left(\mathrm{e}^{2 i \sigma_{L}}-1\right) P_{L}(\cos \theta)=-2 i \eta \frac{\mathrm{e}^{2 i \sigma_{0}}}{1-\cos \theta} \mathrm{e}^{-i \eta \ln \left(\frac{1-\cos \theta}{2}\right)}$,

and $f_{M M}$ is the amplitude generated by the inclusion of the MM interaction

$$
\begin{aligned}
f_{M M}(\theta)= & \frac{\sqrt{4 \pi}}{k} \sum_{L>L_{\max }}^{\infty} \sum_{L^{\prime}>L_{\max }}^{\infty} \sum_{J} \sqrt{2 L+1}(L 0 S \nu \mid J \nu)\left(L^{\prime} M^{\prime} S^{\prime} \nu^{\prime} \mid J \nu\right) \\
& \times \exp \left[i\left(\sigma_{L}+\sigma_{L^{\prime}}-2 \sigma_{0}\right)\right]^{J} T_{L L^{\prime}}^{S S^{\prime}} Y_{L^{\prime} M^{\prime}}(\theta, 0) \\
= & f_{\mu \mu^{\prime}}^{S S^{\prime}}\left[\frac{\cos \theta+2 \mathrm{e}^{-i \eta \ln \left(\frac{1-\cos \theta}{2}\right)}-1}{\sin \theta}-\sum_{L=1}^{L_{\max }} \frac{(2 L+1)}{L(L+1)} \mathrm{e}^{2 i\left(\sigma_{L}-\sigma_{0}\right)} P_{L}^{1}(\cos \theta)\right]
\end{aligned}
$$

with $f_{\mu \mu^{\prime}}^{S S^{\prime}}$ a $6 \times 6$ matrix which take into account the spin structure of the MM interaction [ 5]. In the derivation of the above equation the following relation has been used [ [3, 6]:

$$
\sum_{L=1}^{\infty} \frac{(2 L+1)}{L(L+1)} \mathrm{e}^{2 i \sigma_{L}} P_{L}^{1}(\cos \theta)=\frac{\mathrm{e}^{2 i \sigma_{0}}}{\sin \theta}\left[\cos \theta+2 \mathrm{e}^{-i \eta \ln \left(\frac{1-\cos \theta}{2}\right)}-1\right]
$$

with $P_{L}^{1}$ a generalized Legendre polynomial. The $n-d$ transition matrix is recovered putting $f_{c}=\sigma_{l}=\eta=0$.

If the MM interaction is not considered the sums over $L, L^{\prime}, J$ in the last term of eq.(15) converge very fast due to the finite range of the nuclear interactions. Typically in the low energy region $\left(E_{l a b}<50 \mathrm{MeV}\right)$ states with values of $L, L^{\prime}>10$ can be safely neglected. However when the MM interaction is considered, an infinite number of terms contributes to the construction of the scattering amplitude. In this case the sums on $L, L^{\prime}$ have been divided in two parts depending on a certain value of $L_{\text {max }}$. For $L, L^{\prime} \leq L_{\max }$ the $T$-matrix elements correspond to, and are obtained from, a complete three-body description of the system. For $L, L^{\prime}>L_{\max }$ the centrifugal barrier is sufficiently high to maintain the third particle far from the deuteron and the description of the state can be performed as a two-body system. Moreover, in this regime the nuclear interaction can be neglected and the interaction between the incident nucleon and the deuteron can be considered only as electromagnetic. The T-matrix elements corresponding to values of $L, L^{\prime}>L_{\max }$ have been obtained in Born approximation as

$J_{S S^{\prime}}^{L L^{\prime}}=-k\left(\frac{2 M_{n d}}{\hbar^{2}}\right)<\Omega_{L^{\prime} S^{\prime} J}\left|v_{M M}(N d)\right| \Omega_{L S J}>$

with $M_{N d}$ the $N-d$ reduced mass and $\Omega_{L S J}=F_{L}(k r)\left[Y_{L}(\hat{r}) \otimes \chi_{S}\right]_{J J_{z}}$ is the $N-d$ wave function. Here $F_{L}$ represents the regular Coulomb (Bessel) function for the $p-d(n-d)$ system and $k^{2}=\left(2 M_{n d} / \hbar^{2}\right) E_{c m}$. In the above equation the MM interaction between a nucleon and the deuteron appears explicitly and it is defined as:

$v_{M M}(n d)=-\frac{\alpha}{r^{3}}\left[\frac{\mu_{n} \mu_{d}}{M_{n} M_{d}} S_{n d}^{I}+\frac{\mu_{n}}{2 M_{n} M_{n d}}\left(\mathbf{L} \cdot \mathbf{S}_{n d}+\mathbf{L} \cdot \mathbf{A}_{n d}\right)\right]$ 


$$
\begin{aligned}
v_{M M}(p d)= & -\frac{\alpha}{r^{3}}\left[\frac{\mu_{p} \mu_{d}}{M_{p} M_{d}} S_{p d}^{I}+\left(\frac{\mu_{p}}{2 M_{p} M_{p d}}-\frac{1}{4 M_{p}^{2}}\right)\left(\mathbf{L} \cdot \mathbf{S}_{p d}+\mathbf{L} \cdot \mathbf{A}_{p d}\right)\right. \\
& \left.+\left(\frac{\mu_{d}}{2 M_{d} M_{p d}}-\frac{1}{4 M_{d}^{2}}\right)\left(\mathbf{L} \cdot \mathbf{S}_{p d}-\mathbf{L} \cdot \mathbf{A}_{p d}\right)-\frac{Q_{d}}{2} S_{d}^{I I}\right] \\
S_{N d}^{I}= & 3\left(\mathbf{S}_{N} \cdot \hat{r}\right)\left(\mathbf{S}_{d} \cdot \hat{r}\right)-\mathbf{S}_{N} \cdot \mathbf{S}_{d}, \quad N=n, p \\
S_{d}^{I I}= & 3\left(\mathbf{S}_{d} \cdot \hat{r}\right)^{2}-2
\end{aligned}
$$

where $M_{d}$ is the deuteron mass and $Q_{d}$ is the deuteron quadrupole moment. $\mathbf{S}_{N d}=\mathbf{S}_{N}+\mathbf{S}_{d}$ whereas $\mathbf{A}_{N d}=\mathbf{S}_{N}-\mathbf{S}_{d}$. The deuteron-nucleon distance is $r$ and $\hat{r}$ is the unitary vector giving their relative position.

The $T$-matrix elements of eq.(9) are used to determine the $f_{M M}$ amplitude of eq.(17). They originate a partial wave series which convergences slowly. In fact, after performing the spatial integrals in eq.(9) a term proportional to $\delta_{L L^{\prime}} /[L(L+1)]$ appears that has been summed analytically up to $\infty$ in eq.(17).

\section{N-d observables including MM interaction}

Elastic observables for $N-d$ scattering can be calculated using the transition matrix of eq.(5) using trace operations [ 7]. The calculations presented here have been performed using the complex form of the Kohn variational principle [9] after an expansion of the three-nucleon scattering wave function in terms of the pair correlated hyperspherical harmonic basis [10, 11]. The method used to describe $n-d$ as well as $p-d$ elastic scattering is given in Ref. [8]. The $N N$ interaction we have used is the nuclear part of the AV18 potential with and without the MM interactions defined in eqs.(244). The asymmetric force $\mathbf{L} \cdot \mathbf{A}$ in the $v_{M M}(n p)$ interaction has been considered.

At energies below the deuteron breakup the contribution of the MM interaction is expected to be appreciable. The $n-d$ neutron analyzing power $A_{y}$ has been recently measured at $E_{n}=1.2$ and $1.9 \mathrm{MeV}$ [12]. At these low energies the nuclear part of the transition matrix (last term of eq.(5)) converges already for $L_{\max }=3$. Different theoretical curves have been computed and compared to the experimental data in Fig.1 at $E_{n}=1.2 \mathrm{MeV}$ (left panel) and at $E_{n}=1.9 \mathrm{MeV}$ (right panel). The solid lines correspond to calculations using the AV18 potential and neglecting the MM interaction. These calculations show the usual underestimation that all modern $N N$ forces produces in the description of $A_{y}$. When the MM interaction is taken into account the analyzing powers calculated at both energies, using the last term of eq.(5) up to $L_{\max }=3$, are given by the dashed curves. We can observe a very small influence of the MM interaction in the peak of $A_{y}$ with the tendency of slightly flattening the observable. However, this is an incomplete calculation since the inclusion of the MM interaction requires an infinite number of partial waves in the calculation of the transition matrix. When the MM amplitude $f_{M M}$ is considered the observables are given by the dashed-dotted curves. It is interesting to notice the forward-angle dip structure which already appears in $n-p$ scattering [4]. Only after summing the series up to $\infty$ it is possible to reproduce this particular behavior which is a consequence of the term proportional to $(\cos \theta+1) / \sin \theta$. In fact this term produces a divergence in the $\mathrm{n}$-d differential cross section as $\theta \rightarrow 0$. However this divergence appears at extreme forward angles. In Fig.1 the dip structure is 
only partially shown since $A_{y}=0$ at $\theta=0$. We can conclude that the MM interaction produce a pronounced modification of $A_{y}$ at forward angles but has a very small effect near the peak. Besides the differential cross section and the vector analyzing power other elastic $n-d$ observables as the tensor analyzing powers suffer only minor modifications when the MM interaction is included. The differences are of the order of $1 \%$ or less.
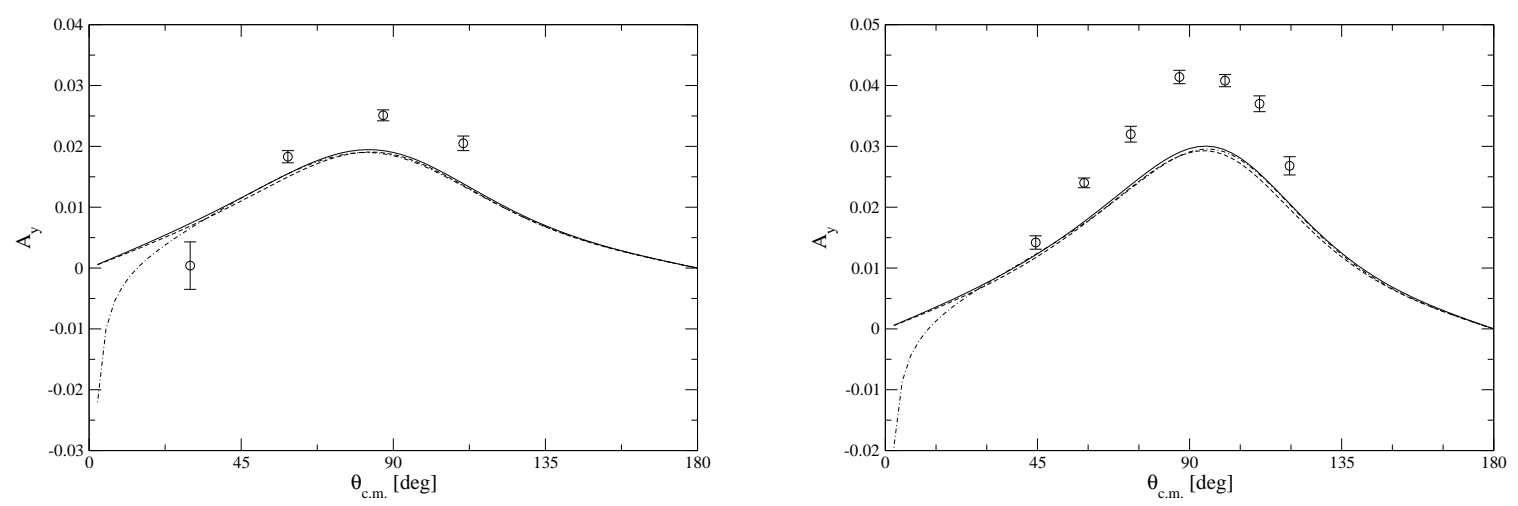

Figure 1. The neutron $A_{y}$ at $E_{n}=1.2 \mathrm{MeV}$ and $1.9 \mathrm{MeV}$. For explanation of the curves see text. Experimental data are from Ref. [12]

For $p-d$ scattering high precision data exist at low energies [ 13, 14, 15, 16, for differential cross sections and vector and tensor analyzing powers. Detailed comparisons to these data has been performed in Refs. [ 13, 14, 17, 18, 19] using AV18 with and without the inclusion of three-body forces. In these studies the Coulomb interaction has been included whereas the MM interaction has not. In order to evaluate the effects of the MM interaction on the vector analyzing powers in presence of the Coulomb field, in Fig.2 we show results at $E_{p}=1$ and $3 \mathrm{MeV}$. Three different calculations have been performed at both energies. The solid line corresponds to the AV18 prediction neglecting the MM interaction. The partial-wave series has been summed up to $L_{\max }=4\left(E_{p}=1 \mathrm{MeV}\right)$ and $L_{\max }=6\left(E_{p}=3 \mathrm{MeV}\right)$. The dashed line corresponds to the same calculation as before but the $T$-matrix elements has been calculated using the AV18+MM potential. The dashed-dotted line corresponds to the complete calculation including the $f_{M M}$ amplitude. We see that the major effect of the MM interaction is obtained around the peak and is appreciable at both energies. There is also an improvement in the description of the observable at forward angles. The observed modifications are due to the interference between the Coulomb and the nuclear plus the MM interaction and not due to high order terms as in the $n-d$ case. In fact high order terms are dominated by the Coulomb interaction and the MM interaction gives a very small contribution. As for $n-d$ scattering, the tensor analyzing powers present very small modifications when the MM interaction is considered. 

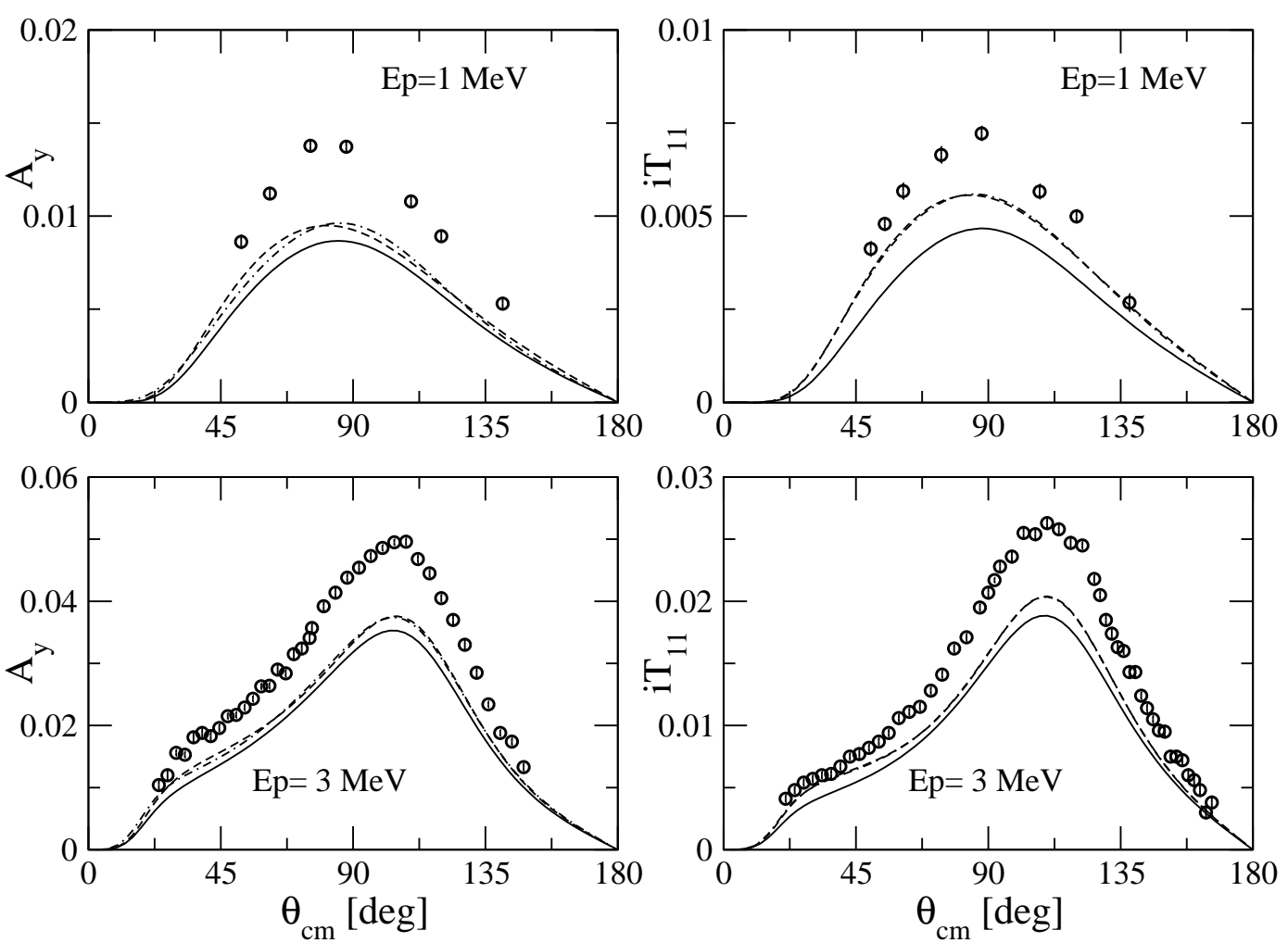

Figure 2. The $p-d$ vector analyzing powers $A_{y}$ and $i T_{11}$. For explanation of the curves see text. Experimental data are from Refs. [14, 16] at $E_{p}=1 \mathrm{MeV}$ and $3 \mathrm{MeV}$ respectively.

Increasing the energy the effect of the MM interaction on $A_{y}$ and $i T_{11}$ diminishes. Above $E_{l a b}=10 \mathrm{MeV}$ calculations using AV18 or AV18+MM give extremely close results [[20]. A different question is the importance of Coulomb effects. In fact, up to $E_{l a b}=30 \mathrm{MeV}$ we can observe appreciable differences in the description of $n-d$ and $p-d$ elastic scattering [ 8. Experimental data are not always conclusive since experiments with neutrons have larger uncertainties than those performed with protons. Several times the description of $p-d$ scattering has been performed using $n-d$ calculations, in particular at high energies [ 21]. To make clear the importance of Coulomb effects as the energy of the collision increases, in Figs.3,4 we show a comparison of $n-d$ and $p-d$ calculations at $E_{l a b}=65 \mathrm{MeV}$. In Fig.3 the differential cross section and $A_{y}$ are shown. Three curves are displayed corresponding to $p-d$ AV18 (solid line), $n-d$ AV18 (dashed line) and $p-d$ AV18+UR (dotted line) and compared to experimental data. In Fig.4 the same calculations are shown for $i T_{11}$ and the three tensor analyzing powers. We can observe appreciable Coulomb effects in $T_{21}$ whereas three-nucleon interaction effects can be observed in the minimum of the differential cross section and in $T_{21}$ and $T_{22}$ as well.

The MM interaction has a different effect in $n-d$ or $p-d$ vector analyzing powers. One 

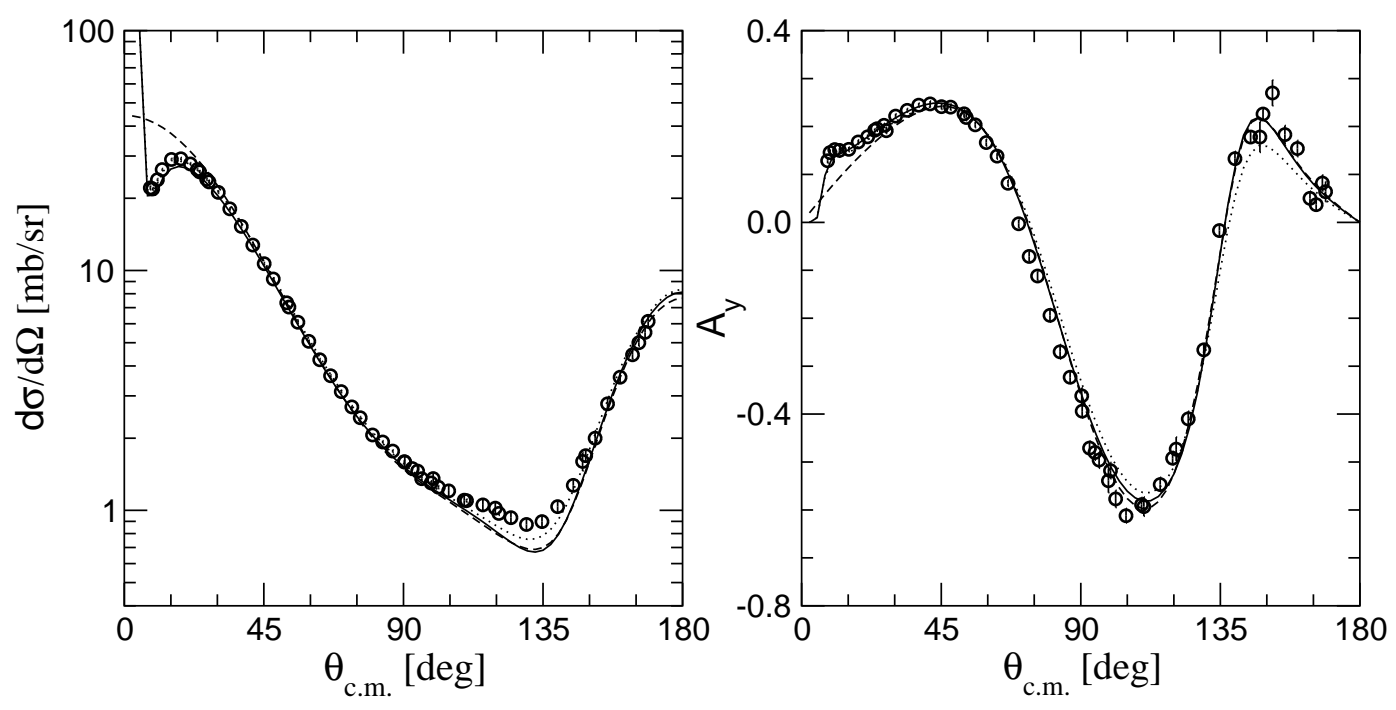

Figure 3. The differential cross section and $A_{y}$ at $E_{l a b}=65 \mathrm{MeV}$. For explanation of the curves see text. Experimental data are from Ref. [22]

reason is the different sign of the neutron and proton magnetic moments. The other reason is the interference with the Coulomb field. However the MM interaction does not help for a better description of the neutron $A_{y}$. On the contrary there is an appreciable improvement in the proton $A_{y}$ as well as in $i T_{11}$, in particular at very low energies. Hence we can study the differences between the experimental data and the calculations at the peak and see if the inclusion of MM interaction helps to clarify a different behavior observed for $n-d$ and $p-d$. In Fig.5 we show the relative difference $\left[A_{y}(\exp )-.A_{y}(t h).\right] / A_{y}(\exp$.$) at the peak$ for $n-d$ and for $p-d$ scattering. In this last case the AV18 and AV18+MM results have been considered. For $n-d$ both results are extremely close at the peak and only the AV18 result has been considered. We see that without the inclusion of the MM interaction the underestimation of the proton $A_{y}$ is much more pronounced than the neutron $A_{y}$. When the MM interaction is considered both, the $n-d$ and $p-d A_{y}$ are predicted with a reduction of similar size (around 25\%) in all the energy interval below $16 \mathrm{MeV}$. Above 16 $\mathrm{MeV}$ the differences on the peak between theory and experiment diminish and practically disappear at $30 \mathrm{MeV}$. In Fig. 5 we see that at $18 \mathrm{MeV}$ the difference has been reduced to $20 \%$.

\section{4. conclusions}

We have shown that it is possible to perform detailed calculations to describe $N-d$ elastic scattering using a potential including the nuclear plus Coulomb plus MM interactions. Though the strength of the MM interaction is small compared to the nuclear one, its long range produces appreciable effects in some observables as the $n-d$ differential 
cross section and vector analyzing powers. For $p-d$ scattering we observed an increase in vector analyzing powers at low energies. The importance of Coulomb effects has been analyzed in Ref. [8] below $30 \mathrm{MeV}$. Here we have shown that at $65 \mathrm{MeV}$ Coulomb effects are very reduced in most of the elastic observables with the exception of $T_{21}$ where an appreciable effect has been observed in the peak.

In the low energy regime the electromagnetic potential produces modifications in the observables that are of similar size to those ones produced by the most frequently used three-nucleon interactions, as the UR potential. Therefore when the three-nucleon continuum would be used to study the structure of the three-nucleon interaction, the complete electromagnetic NN potential should be taken into account.
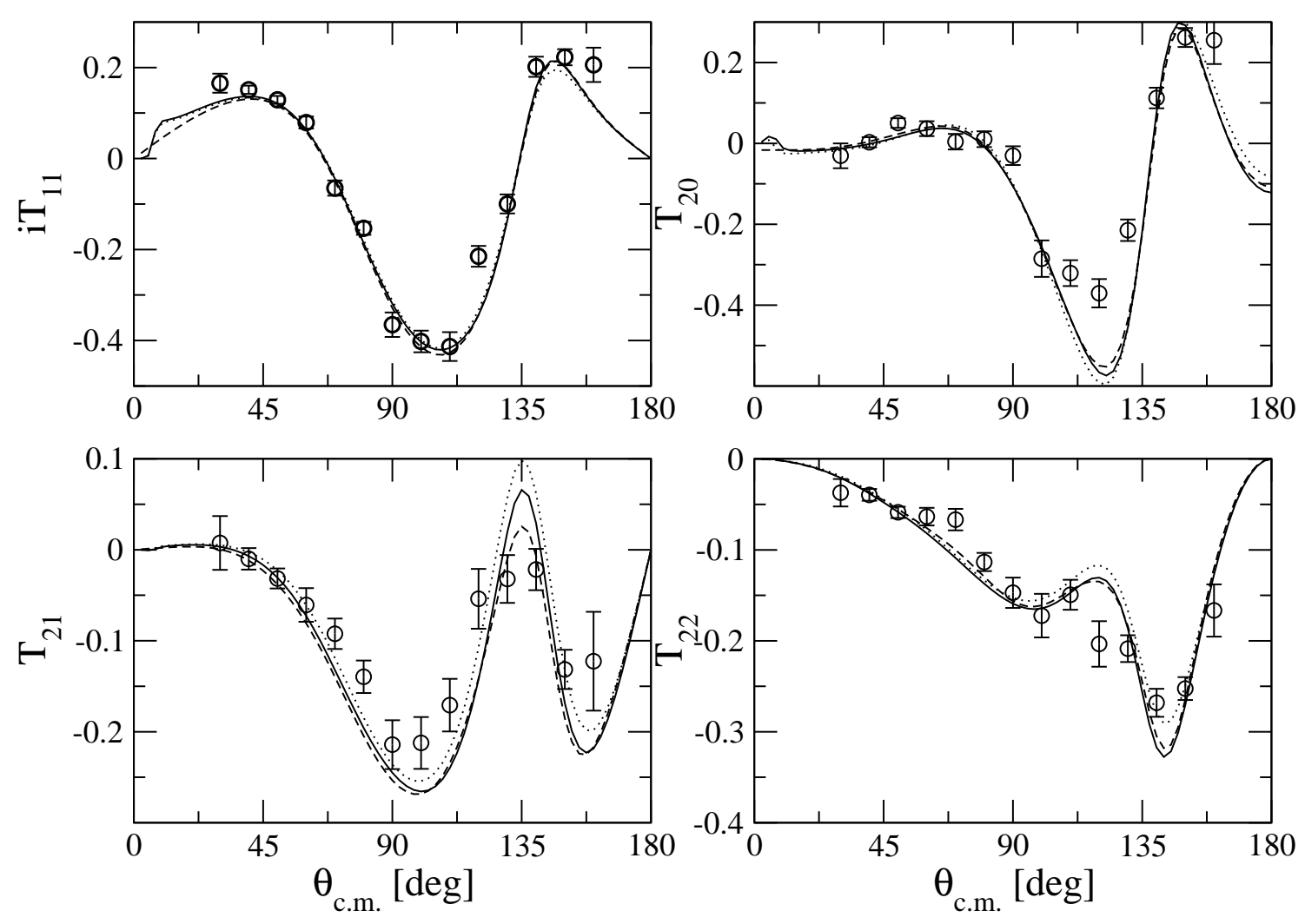

Figure 4. The deuteron analyzing power $i T_{11}$ and the tensor analyzing powers $T_{20}, T_{21}, T_{22}$ at $E_{l a b}=65 \mathrm{MeV}$. For explanation of the curves see text. Experimental data are from Ref. [23] 


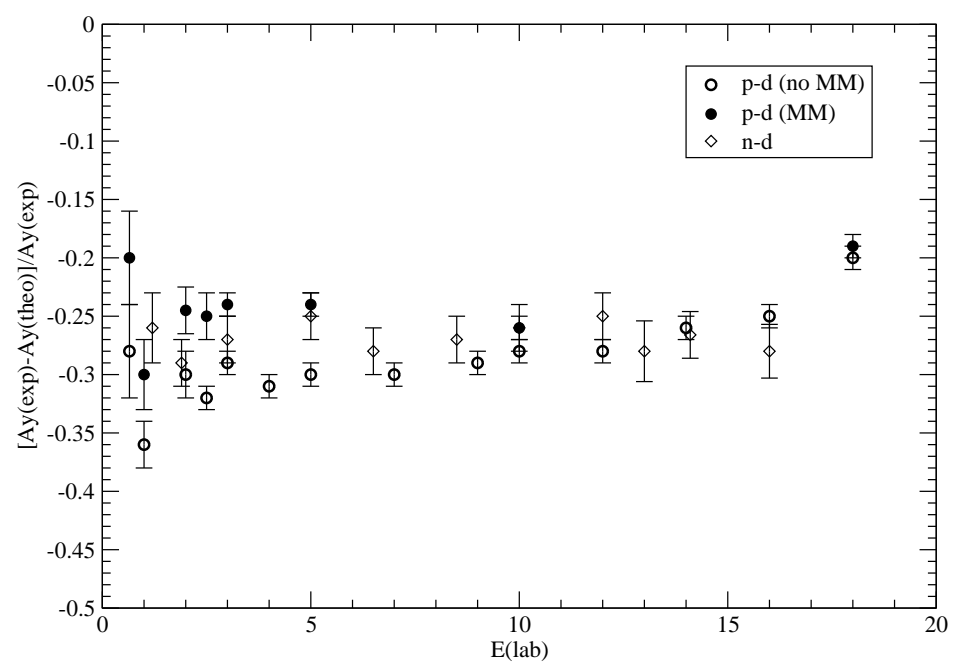

Figure 5. Relative difference at the peak of $A_{y}$ as a function of energy.

\section{REFERENCES}

1. R.B. Wiringa, V.G.J. Stoks, R. Schiavilla, Phys. Rev. C51 (1995) 38

2. B.S. Pudliner et al., Phys. Rev. Lett. 74 (1995) 4396

3. L.D. Knutson and D. Chiang, Phys. Rev. C (1978) 1958

4. V.G.J. Stoks and J.J. de Swart, Phys. Rev. C (1990) 1235

5. A. Kievsky, M. Viviani, L. Marcucci and S. Rosati, in preparation

6. L.D. Knutson, private communication

7. R.G. Seyler, Nucl. Phys. A124 (1969) 253

8. A. Kievsky, M. Viviani and S. Rosati, Phys. Rev. C64 (2001) 024002

9. A. Kievsky, Nucl. Phys. A624 (1997) 125

10. A. Kievsky, M. Viviani, S. Rosati, Nucl. Phys. A551 (1994) 241

11. A. Kievsky, M. Viviani, S. Rosati, Nucl. Phys. A577 (1994) 511

12. E.M. Neidel et al., Phys. Lett. B552 (2003) 29

13. C.R. Brune et al., Phys. Rev. C63 (2001) 044013

14. M.H. Wood et al., Phys. Rev. C65 (2002) 034002

15. L.D. Knutson, L.O. Lamm and J.E. McAninch, Phys. Rev. Lett.71 (1993) 3762

16. S. Shimizu et al., Phys. Rev. C52 (1995) 1193

17. A. Kievsky et al., Phys. Rev. C63 (2001) 024005

18. A. Kievsky, S. Rosati, W. Tornow and M. Viviani, Nucl. Phys. A607 (1996) 402

19. A. Kievsky, M. Viviani and S. Rosati, Phys. Rev. C52 (1995) R15

20. A. Kievsky, M. Viviani, L.E. Marcucci and S. Rosati, Few-Body Syst. Suppl. 14 (2003) 111

21. H. Witala et al., Phys. Rev. C63 (2001) 024007

22. H. Shimizu et al., Nucl. Phys. A382 (1982) 242

23. H. Witala et al., Few-Body Syst. 15 (1993) 67 